\title{
A possible optical counterpart to the old nearby pulsar J0108-1431`
}

\author{
R. P. Mignani ${ }^{1}$, G. G. Pavlov ${ }^{2}$, and O. Kargaltsev ${ }^{2,3}$ \\ 1 Mullard Space Science Laboratory, University College London, Holmbury St. Mary, Dorking, Surrey, RH5 6NT, UK \\ e-mail: rm2@mssl.ucl.ac.uk \\ 2 Department of Astronomy and Astrophysics, Pennsylvania State University, PA 16802, USA \\ e-mail: pavlov@astro.psu.edu \\ 3 Department of Astronomy, University of Florida, 32611, USA \\ e-mail: oyk100@astro.ufl.edu
}

Received 16 May 2008 / Accepted 1 July 2008

\begin{abstract}
Context. The multi-wavelength study of old ( $>100 \mathrm{Myr}$ ) radio pulsars holds the key to understanding the long-term evolution of neutron stars, including the advanced stages of neutron star cooling and the evolution of the magnetosphere. Optical/UV observations are particularly useful for such studies because they allow one to explore both thermal and non-thermal emission processes. In particular, studying the optical/UV emission constrains the temperature of the bulk of the neutron star surface, too cold to be measured in X-ray observations.

Aims. Aim of this work is to identify the optical counterpart of the very old (166 Myr) radio pulsar J0108-1431.

Methods. We have re-analyzed our original Very Large Telescope (VLT) observations, where a very faint object was tentatively detected close to the radio position, near the edge of a field galaxy.

Results. We found that the backward extrapolation of the PSR J0108-1431 proper motion recently measured by Chandra fits the position of this object. Based on that, we propose it as a viable candidate for the optical counterpart to PSR J0108-1431. The object fluxes $\left(U=26.4 \pm 0.3 ; B \approx 27.9 ; V \geq 27.8\right.$ ) are consistent with a thermal spectrum with a brightness temperature of $\sim 9 \times 10^{4} \mathrm{~K}$ (for $R=13 \mathrm{~km}$ at a distance of $130 \mathrm{pc}$ ), emitted from the bulk of the neutron star surface.

Conclusions. New optical observations are required to confirm the optical identification of PSR J0108-1431 and measure its spectrum.
\end{abstract}

Key words. astrometry - stars: pulsars: individual: PSR J0108-1431

\section{Introduction}

The radio pulsar J0108-1431 was discovered by Tauris et al. (1994) during the Parkes Southern Pulsar Survey (Manchester et al. 1996). PSR J0108-1431 is one of the closest pulsars. Its dispersion measure of $2.38 \mathrm{pc} \mathrm{cm}^{-3}$ (D'Amico et al. 1998), the smallest known for a radio pulsar, corresponds to a distance of $130 \mathrm{pc}$, according to the Galactic electron density model by Taylor \& Cordes (1993) (180 pc in the model by Cordes \& Lazio 2002). The pulsar's period, $P=0.808 \mathrm{~s}$, and period derivative, $\dot{P}=7.44 \times 10^{-17} \mathrm{~s} \mathrm{~s}^{-1}$ (D'Amico et al. 1998), correspond to the spin-down age $\tau=P / 2 \dot{P}=166 \mathrm{Myr}$, rotational energy loss rate $\dot{E}=5.8 \times 10^{30} \mathrm{erg} \mathrm{s}^{-1}$, and surface magnetic field $B=2.5 \times 10^{11}$ G. PSR J0108-1431 is thus one of the oldest non-recycled radio pulsars known to date. In particular, its position in the $P-\dot{P}$ diagram falls quite close to the pulsar death line. Indeed, with a $400 \mathrm{MHz}$ luminosity of $0.15 d_{130}^{2} \mathrm{mJy} \mathrm{kpc}^{2}$, where $d_{130}$ is the distance in units of $130 \mathrm{pc}$, PSR J0108-1431 is the second faintest radio pulsar.

Soon after its discovery, there were several attempts to detect PSR J0108-1431 at other wavelengths. In X-rays, the pulsar was not detected in the ROSAT All Sky Survey, while pointed UV,

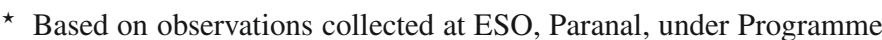
65.H-0400(A)
IR, and optical observations with EUVE (Korpela \& Bowyer 1998), ISO (Koch-Miramond et al. 2002), and the SAO RAS $6 \mathrm{~m}$ telescope (Kurt et al. 2000) did not reveal candidate counterparts. Mignani et al. (2003) measured the most accurate radiointerferometric position with ATCA and performed deep optical observations with the Very Large Telescope (VLT), but they did not propose a candidate counterpart. Recently, PSR J0108-1431 was identified in a $30 \mathrm{ks}$ exposure with the Chandra ACIS detector (Pavlov et al. 2008), based on its position close to the ATCA radio coordinates and on the high $(>300) \mathrm{X}$-ray-to-optical flux ratio deduced with the aid of the VLT images of Mignani et al. (2003). The pulsar's spectrum can be fitted with either a powerlaw with photon index $\Gamma \approx 2.2$ (for the hydrogen column density $N_{\mathrm{H}}=7.3 \times 10^{19} \mathrm{~cm}^{-2}$ estimated from the pulsar's dispersion measure) or a blackbody with temperature $k T \approx 0.28 \mathrm{keV}$ and emitting area of $\sim 50 d_{130}^{2} \mathrm{~m}^{2}$. The estimated $0.3-8 \mathrm{keV} \mathrm{lu}-$ minosity, $\sim 10^{28} d_{130}^{2} \mathrm{erg} \mathrm{s}^{-1}$, translates into the X-ray emitting efficiency $\eta_{\mathrm{X}} \equiv L_{\mathrm{X}} / \dot{E} \sim 4 \times 10^{-3} d_{130}^{2}$, somewhat higher than for most other pulsars. The comparison between the Chandra and ATCA coordinates of PSR J0108-1431 yielded its first proper motion measurement $\left(199 \pm 65 \mathrm{mas} \mathrm{yr}^{-1}\right)$, corresponding to a transverse velocity of $129 \pm 42 d_{130} \mathrm{~km} \mathrm{~s}^{-1}$ (Pavlov et al. 2008).

In this paper we present a re-analysis of the VLT observations of Mignani et al. (2003). The analysis and results are described in Sect. 2, while the discussion is presented in Sect. 3. 


\section{Observations and data analysis}

Optical observations of the PSR J0108-1431 field were performed in July and August 2000 with the VLT, using the original version of the FOcal Reducer/low dispersion Spectrograph (FORS1). At the time of the observations the instrument was equipped with a four-port $2048 \times 2084$ CCD with a 0.2 pixel size and a field of view of $6 ! 8 \times 6.8$ in its standard resolution mode. Observations were performed in the Bessel $U, B$ and $V$ filters (see Mignani et al. 2003, for a detailed description of the observations and data reduction). As discussed by Mignani et al. (2003), the identification of the PSR J0108-1431 counterpart was complicated by the fact that the ATCA radio position was close to the edge of a relatively bright elliptical field galaxy. A possible counterpart was noticed in the $U$ and $B$ bands only, $\approx 0^{\prime} .3$ northwest from to the radio position. However, the marginal detection significance of this object and its proximity to the galaxy made it difficult to establish whether it was a genuine detection or a background enhancement, perhaps associated with the galaxy. For this reason, no identification with the pulsar was claimed.

\subsection{Astrometry}

The recent pulsar proper motion measurement by Pavlov et al. (2008) prompted us to re-investigate the possibility that PSR J0108-1431 can be identified with the object marginally detected in the VLT data. We recomputed the astrometric calibration of our VLT/FORS1 images using as a reference the updated release of the GSC2 (ver. 3.2), which provided a larger number of reference stars in the field and an improved coordinate accuracy, with a mean random radial error (for stellar objects) $\sigma_{\mathrm{GSC}}=0.3$ (Lasker et al. 2008). The detector coordinates of 20 selected GSC2 stars (all non-saturated, with a well-defined PSF, and evenly distributed in the field of view) were measured by fitting a Gaussian function to their intensity profiles using the Graphical Astronomy and Image Analysis (GAIA) tool ${ }^{1}$. The coordinate transformation between the detector and the celestial reference frame was then computed using the Starlink package ASTROM ${ }^{2}$. The rms of our astrometric fit was 0.14 for each of the two coordinates, corresponding to the radial error $\sigma_{\text {fit }}=0.21$. The new astrometric solution is consistent with the one obtained by Mignani et al. (2003), but it is a factor of 2 more precise, thanks to the larger number of GSC2 stars and to the improved astrometric accuracy. We estimated the overall uncertainty of our astrometric solution by adding in quadrature the $\sigma_{\text {fit }}$ and the uncertainty $\sigma_{\text {tr }}=\sqrt{3 / N_{\mathrm{s}}} \sigma_{\mathrm{GSC}}=0.12$ with which we can register our field on the GSC2 reference frame $\left(N_{\mathrm{s}}=20\right.$ is the number of reference stars, and $\sqrt{3}$ accounts for the free parameters in the astrometric fit; e.g., Lattanzi et al. 1997). The uncertainty on the reference star centroids is below 0.'01 and was neglected. After accounting for the $0{ }^{\prime} .15$ mean systematic uncertainty on the tie of the GSC2 to the International Celestial Reference Frame (Lasker et al. 2008), the overall uncertainty of our absolute astrometry is 0.28 ( $0 . ' 19$ per coordinate).

The ATCA and Chandra positions of PSR J0108-1431 are shown in Fig. 1, overlayed on the FORS1 $U$ band image after astrometric recalibration. As seen from Fig. 1, the backward proper motion extrapolation closely fits the position of the object tentatively detected at the edge of the field galaxy. This is more quantitatively shown in Fig. 2, where we compare the relative

\footnotetext{
1 http://star-www. dur . ac . uk pdraper/gaia/gaia.html

2 http://star-www.rl.ac.uk/Software/software.htm
}

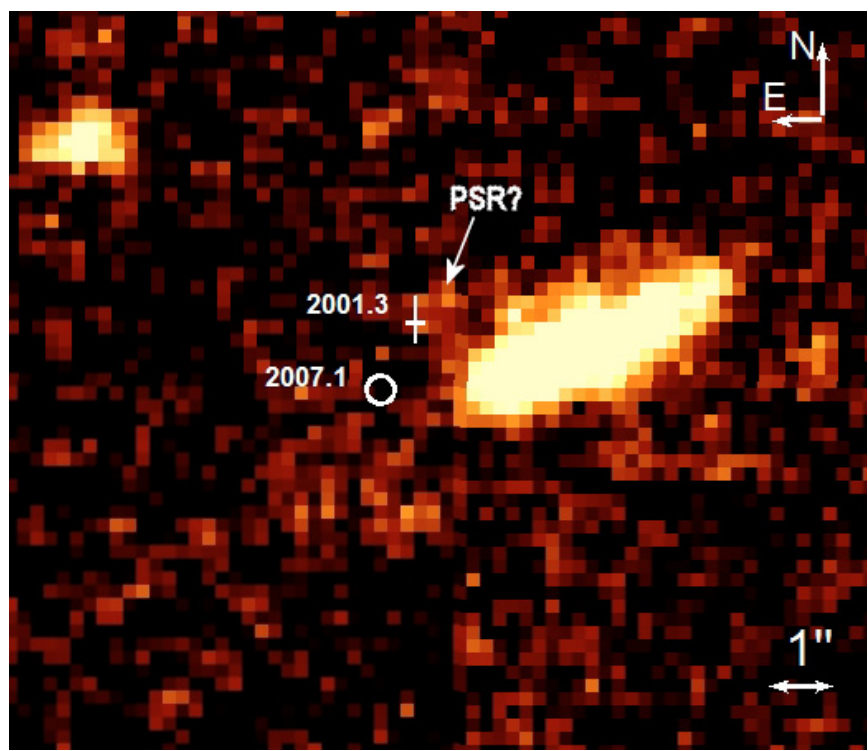

Fig. 1. VLT $U$ band image of the PSR J0108-1431 field obtained by Mignani et al. (2003). The pulsar candidate counterpart $(U=26.4 \pm 0.3)$ is marked by an arrow. The cross shows the radio-interferometric position of Mignani et al. (2003) while the circle corresponds to the most recent pulsar position obtained with Chandra by Pavlov et al. (2008). The position of the candidate pulsar optical counterpart (epoch 2000.6) closely fits the backward proper motion extrapolation.

offsets between the coordinates of the optical source and those of PSR J0108-1431 measured by ATCA and Chandra. The coordinates of the optical source, computed with our astrometric solution, are $\alpha=01^{\mathrm{h}} 08^{\mathrm{m}} 08^{\mathrm{s}} .301$ and $\delta=-14^{\circ} 31^{\prime} 49^{\prime} \cdot 15$, (J2000), with the $1 \sigma$ uncertainty of 0.27 in each coordinate. This value includes the overall uncertainty of our absolute astrometry and the object centroiding error, added in quadrature. Since it is practically impossible to fit a PSF to the object profile, we assumed a conservative estimate of \pm 1 pixel $\left( \pm 0^{\prime} .2\right)$ for the centroiding error.

The yearly displacement computed using all the available coordinates yields the proper motion of $\mu_{\alpha}=100 \pm 41 \mathrm{mas} \mathrm{yr}^{-1}$,

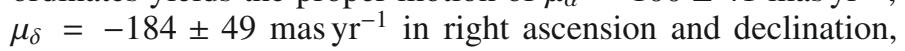
respectively. These proper motion values are in excellent agreement with those reported by Pavlov et al. (2008).

The probability of a chance coincidence between the position of the candidate counterpart and the position computed from the backward extrapolation of the pulsar's proper motion (Pavlov et al. 2008) can be estimated as $P=1-\exp \left(-\pi \sigma r^{2}\right)$, where $r$ is the matching radius $\left(0 .{ }^{\prime} 6\right.$, taking account of the uncertainties of the Chandra position and the backward proper motion extrapolation), and $\sigma$ is the density of stellar objects in the pulsar vicinity. For starlike objects (ellipticity $e<0.2$ ) with magnitudes $U>25$ (a conservative a priori upper limit on the actual pulsar brightness; see Sect. 3) and detected with at least $3 \sigma$ significance (to filter out background fluctuations), we found $\sigma=0.001 \operatorname{arcsec}^{-2}$, which gives $P=1.1 \times 10^{-3}$, low enough to claim the association of the pulsar with the candidate counterpart. Thus, based on the agreement between its position and the proper motion extrapolation, we propose the object originally identified in the VLT images of Mignani et al. (2003) as the candidate optical counterpart of PSR J0108-1431. 

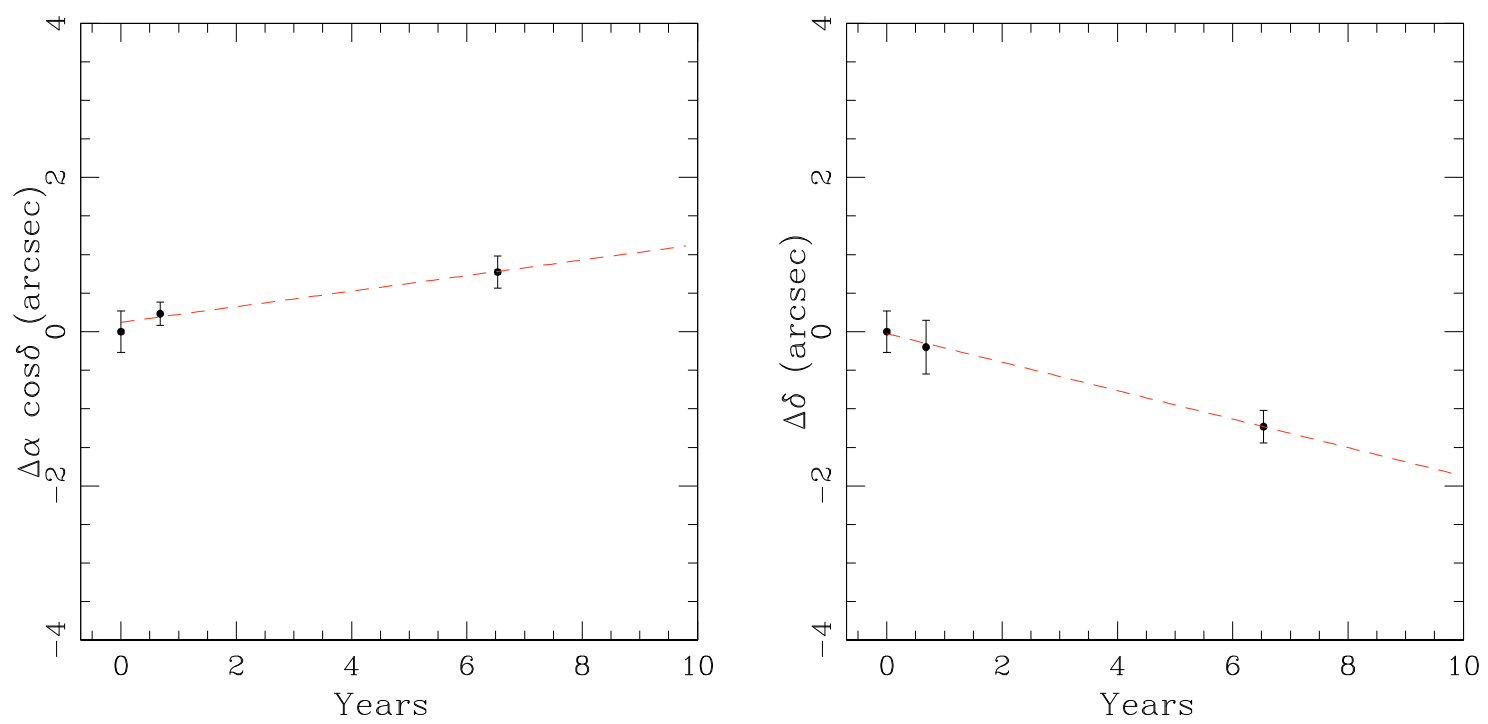

Fig. 2. Proper motion measurement in right ascension (left) and declination (right) computed from the optical (VLT), radio (ATCA), and X-rays (Chandra) coordinates of PSR J0108-1431. The displacements are computed with respect to the first epoch VLT position of the counterpart candidate.

\subsection{Photometry}

We carefully remeasured the flux of the candidate counterpart. The object is fairly well detected in the $U$ band $(\approx 5 \sigma)$, but it is only marginally detected in the $B$ band $(\approx 3 \sigma)$. Due to the difficulties in fitting a model PSF to the object profile, we measured its flux through aperture photometry. Sky background was sampled in a number of regions selected within a radius of $\sim 4^{\prime \prime}$ from the object (far enough from the field galaxy and from other relatively bright objects in the vicinity), with sizes 2 to 4 times larger than the FWHM of the image PSF. In order to increase as much as possible the signal-to-noise ratio, photometry was computed using customized aperture diameters of $0 .{ }^{\prime} 6$ and 0.7 , i.e. about the size of the image PSF in the $B$ and $U$-band, respectively. Due to the uncertainty in the determination of the object centroiding (see Sect. 2.1), measurements were iterated centering the aperture at different positions around the best centroiding estimate. Results were found consistent within the measurement uncertainties. Aperture correction was then applied using as a reference the fitted growth curves of well-suited reference stars selected in the field. Airmass correction was applied using the Paranal extinction coefficients measured with FORS1. Photometric calibration was applied using the mean, extinction corrected, night zero points ${ }^{3}$.

We obtained $U=26.4 \pm 0.3$ and $B \approx 27.9$. As the higher background in the $B$ band caused by the close-by galaxy makes our measurement very uncertain, we will use a fiducial error \pm 0.5 for the $B$ magnitude. The object is undetected in the $V$ band. We have remeasured the flux upper limit at the location of the candidate counterpart and obtained $V>27.8$ at a $3 \sigma$ level, consistent with the limit reported by Mignani et al. (2003). The derived spectral fluxes are shown in Fig. 3. Since the $N_{\mathrm{H}}=7.3 \times 10^{19} \mathrm{~cm}^{-2}$, estimated from the pulsar's dispersion measure, implies a reddening of $\approx 0.05 \mathrm{mag}$, i.e. much smaller than our photometric errors, we neglected the interstellar extinction correction.

\footnotetext{
${ }^{3}$ http://www.eso.org/observing/dfo/quality/FORS1/qc/ qc1.html
}

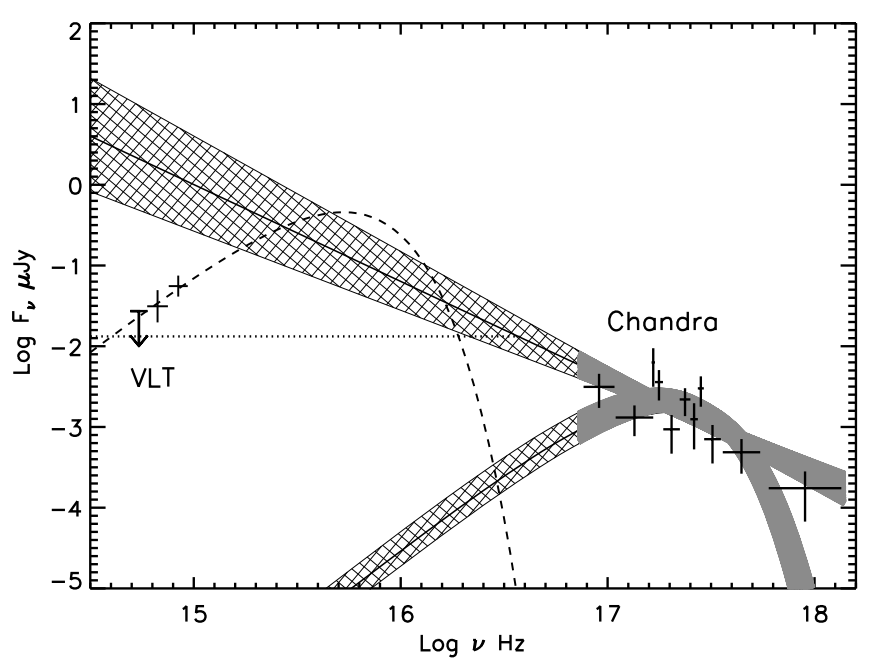

Fig. 3. Results of the optical photometry of the PSR J0108-1431 candidate counterpart ( $U$ and $B$ band fluxes and $V$ band upper limit) and the X-ray spectrum of PSR J0108-1431 measured with Chandra in the $0.3-8 \mathrm{keV}$ band (Pavlov et al. 2008). The fits of the X-ray spectrum with the power-law and blackbody models are shown, with their extrapolations to lower energies. The hatched areas correspond to $1 \sigma$ uncertainties of the X-ray fits. The dashed curve shows a blackbody spectrum with the temperature of $9 \times 10^{4} \mathrm{~K}$ (for $R=13 \mathrm{~km}, d=130 \mathrm{pc}$ ). The dotted line shows an example of a flat power-law additional component in the optical- $U V$ band, compatible with the measured $V$ band upper limit.

\section{Discussion}

With only one fairly accurate measurement, our photometry does not allow us to determine the spectral slope for the candidate counterpart. The measured optical fluxes are compatible with a thermal (Rayleigh-Jeans) spectrum emitted from the bulk of the neutron star surface with the (brightness) temperature $T=(7-10) \times 10^{4}\left(d_{130} / R_{13}\right)^{2} \mathrm{~K}$, where $R_{13}$ is the apparent neutron star radius in units of $13 \mathrm{~km}$ as seen from infinity. An 
example of the blackbody spectrum consistent with the optical fluxes of the candidate counterpart is shown in Fig. 3.

The $U$ and $B$ band fluxes and the $V$ band upper limit cannot be described by a flat or decreasing power-law spectrum, $F_{v} \propto v^{-\alpha}$ with $\alpha \geq 0$, such as is expected for magnetospheric emission. It could be described by an increasing power-law, but power-laws with $\alpha<0$ have not been observed so far in the optical-UV spectra of rotation-powered neutron stars (see, e.g., Mignani et al. 2007). Thus, it is unlikely that the optical emission is purely magnetospheric. Of course, we cannot rule out that a magnetospheric spectral component is present at a level below the $V$ band limit (Fig. 3).

The optical spectral fluxes are inconsistent with the extrapolations of the power-law and blackbody X-ray fits (Fig. 3). In the thermal interpretation, the X-ray emission comes from small, hot polar caps, whose contribution to the optical spectrum is negligible, but the optical emission may come from the bulk of the neutron star surface, which is too cold to be seen in X-rays. If the X-ray spectrum is predominantly magnetospheric, then the optical through X-ray spectrum of the magnetospheric emission cannot be described by a simple power-law, but it could be described by a model in which the spectral slopes are different in the X-rays and optical (e.g., $\alpha_{\mathrm{X}}=1.2, \alpha_{\mathrm{O}}=0$; see Fig. 3 for an example).

If further observations confirm that the object is the pulsar counterpart with a predominantly thermal optical-UV spectrum (as suggested by Fig. 3), this would have important implications for understanding the thermal evolution of old neutron stars and the processes in their interiors. Current neutron star cooling models are rather uncertain for ages greater than 1 Myr because the effect of possible (re)heating processes is unclear. Such processes include the Joule heating, heating caused by readjustment of the equilibrium neutron star structure in the course of its evolution, or heating caused by the effects of superfluidity in the neutron star interiors (see Fernàndez \& Reisenegger 2005, and Schaab et al. 1999, for a review). Evidence for reheating has been found in the much older recycled pulsar J0437-4715, which showed a brightness temperature of $\sim 1 \times 10^{5} \mathrm{~K}$ (Kargaltsev et al. 2004). The measurement of a similar temperature in PSR J0108-1431 would mean that some heating processes operate in slowly rotating, non-recycled pulsars. (We should note, however, that knowing the distance to the pulsar would be necessary to measure the surface temperature from the flux measurements in the Rayleigh-Jeans tail, so that measuring the parallax of PSR J0108-1431 is badly needed.)

If further observations find a non-thermal component in the optical spectrum, this would be important for understanding the long-term evolution of the rotation-powered magnetospheric emission. Recent observations indicate that even very old pulsars are still efficient magnetospheric emitters, with an even higher fraction of their spin-down power emitted in the X-ray and optical bands than in younger pulsars (Kargaltsev et al. 2006; Zharikov et al. 2006; Pavlov et al. 2008). In particular, in the optical/UV this is demonstrated by the cases of the pulsars
B1929+10 (Mignani et al. 2002) and B0950+08 (Zharikov et al. 2004), which exhibit flat optical spectra with optical efficiencies $\eta_{\mathrm{O}} \equiv L_{\mathrm{O}} / \dot{E} \sim 1 \times 10^{-6}$ and $\sim 4 \times 10^{-6}$, respectively (Zavlin \& Pavlov 2004). If at least part of the detected emission from the pulsar counterpart candidate were of a magnetospheric origin, then the optical efficiency (e.g., $\eta_{\mathrm{O}} \sim 2 \times 10^{-5} d_{130}^{2}$ for the flat spectrum shown by the dotted line in Fig. 3) would be rather high compared to the younger pulsars B1929+10 and B0950+08. On the other hand, the (distance-independent) ratio of the $\mathrm{X}$-ray and optical efficiencies $\left(\eta_{\mathrm{O}} / \eta_{\mathrm{X}} \sim 0.005\right.$ for the above example) is within the range found for other pulsars detected in the optical (see Fig. 6 of Zavlin \& Pavlov 2004).

\section{Summary}

By re-analyzing the VLT images of Mignani et al. (2003) we have identified a posteriori a possible optical counterpart to PSR J0108-1431, based on its positional consistency with the backward pulsar proper motion extrapolation. If our identification is correct, this would be the tenth (or eleventh, if the detection of PSR B1133+16 by Zharikov et al. 2008, is confirmed) rotation-powered pulsar detected in the optical/UV. In any case, PSR J0108-1431 would be the oldest non-recycled radio pulsar identified at optical wavelengths. As almost 8 years have passed since the reported observations, the optical identification could be easily confirmed by proper motion measurement of the candidate counterpart in deep optical/UV observations.

Acknowledgements. R.P.M. acknowledges STFC for support through a Rolling Grant and the ESO/Chile Science Visitor Programme for supporting his visit at the Santiago offices, where this work was carried out.

\section{References}

Cordes, J. M., \& Lazio, T. J. W. 2002, [arXiv: astro-ph/0207156] D’Amico, N., Stappers, B. W., Bailes, M., et al. 1998, MNRAS, 297, 28 Fernàndez, R., \& Reisenegger, A. 2005, ApJ, 625, 291

Kargaltsev, O., Pavlov, G. G., \& Romani, R. W. 2004, ApJ, 602, 327

Kargaltsev, O., Pavlov, G. G., \& Garmire, G. P. 2006, ApJ, 636, 436 Koch-Miramond, L., Haas, M., Pantin, E., et al. 2002, A\&A, 387, 233 Korpela, E. J., \& Bowyer, S. 1998, AJ, 115, 2551

Kurt, V. G., Komarova, V. N., Fatkhullin, T. A., et al. 2000, Bull. Spec. Astrophys. Obs., 49, 5

Lasker, B. M., et al. 2008, AJ, submitted

Lattanzi, M. G., Capetti, A., \& Macchetto, F. D. 1997, A\&A, 318, 997

Mignani, R. P., De Luca, A., Caraveo, P. A., \& Becker, W. 2002, ApJ, 580, 147

Mignani, R. P., Manchester, R. N., \& Pavlov, G. G. 2003, ApJ, 582, 97

Mignani, R. P., Zharikov, S., \& Caraveo, P. A. 2007, A\&A, 473, 891

Pavlov, G. G., Kargaltsev, O., Wong, J. A., \& Garmire, G. P. 2008, ApJ, submitted [arXiv:0803.0761]

Schaab, Ch., Sedrakian, A., Weber, F., \& Weigel, M. K. 1999, A\&A, 346, 465

Tauris, T. M., Nicastro, L., Johnston, S., et al. 1994, ApJ, 428, L53

Taylor, J. H., \& Cordes, J. M. 1993, ApJ, 411, 674

Zavlin, V. E., \& Pavlov, G. G. 2004, ApJ, 616, 452

Zharikov, S. V., Shibanov, Yu. A., Mennickent, R. E., Komarova, V. N., \& Tovmassian, G. H. 2004, A\&A, 417, 1017

Zharikov, S. V., Shibanov, Yu. A., \& Komarova, V. N. 2006, AdSpR, 37, 1979

Zharikov, S. V., Shibanov, Yu. A., Mennickent, R. E., \& Komarova, V. N. 2008, A\&A, 479, 793 